\title{
Effects of preoperative, scheduled administration of antiemetics in reducing postoperative nausea and vomiting in patients undergoing total knee arthroplasty
}

Hyung Jun Park, MD, MMSc${ }^{a}$, Moon Jong Chang, MD, PhD ${ }^{a}{ }^{*}$ (D), Seung-Baik Kang, MD, PhDa ${ }^{a}$-ung Hwang, $\mathrm{MD}, \mathrm{PhD}^{\mathrm{b}}$, Jong Seop Kim, MD, BS${ }^{\mathrm{a}}$, Chong Bum Chang, $\mathrm{MD}, \mathrm{PhD}^{\mathrm{c}}$

\begin{abstract}
There is no established protocol regarding the timing of administration of antiemetics in patients undergoing total knee arthroplasty (TKA). The purpose of this study was to determine whether preoperative, rather than postoperative administration of an antiemetic could reduce postoperative nausea and vomiting (PONV) in patients undergoing TKA, and whether there was a difference in postoperative pain, patient satisfaction and complications after TKA between the 2 different administration times.

The included patients $(N=101)$ either received intravenous administration of the ramosetron 1 hour before surgery $(N=50)$ or at the end of surgery $(\mathrm{N}=51)$ consecutively order. The incidence of PONV and the frequency of rescue medicine use were recorded until 48 hours postoperatively. The severity of postoperative pain and patient satisfaction were assessed using the visual analogue scale. The incidence of complications associated with use of antiemetic was assessed.

Preoperative administration of ramosetron did not decrease PONV during the first 48 hours. There was no significant difference in the incidence of nausea and vomiting, use of rescue antiemetics, and the severity of nausea $(P>.05)$. Postoperative pain, satisfaction scores, and the incidence of complications were not different between the 2 groups $(P>.05)$.

Preoperative administration of ramosetron did not show clinical advantage in reducing POVN, postoperative pain and improving patient satisfaction. However, the outcomes of complications were not inferior to those of postoperative administration. Therefore, under the current protocol of multimodal therapies, timing of administration of pre-emptive antiemetic did not have significant effect on PONV.
\end{abstract}

Abbreviations: $\mathrm{LOS}=$ length of stay, $\mathrm{PAI}=$ periarticular injection, $\mathrm{PCA}=$ patient-controlled analgesia, $\mathrm{PONV}=$ postoperative nausea and vomiting, TKA = total knee arthroplasty, VAS = visual analogue scale.

Keywords: antiemetics, PONV, TKA

\section{Introduction}

Postoperative nausea and vomiting (PONV) is a relatively common yet disconcerting side effect in patients undergoing total knee arthroplasty (TKA). ${ }^{[1]}$ The incidence of PONV after TKA has been reported to be between $20 \%$ and $83 \% .^{[2,3]}$ Prolonged PONV after surgery causes poor oral intake, delayed patient recovery, and an increase in the length of hospital stay (LOS). ${ }^{[4]}$ Furthermore, PONV is associated with patient dissatisfaction after $\mathrm{TKA}^{[5,6]}$. Therefore, it is important to understand the management of PONV in patients undergoing TKA. Traditionally, antiemetics have been used depending on the patients needs after surgery. However, post-surgical administration of antiemetics alone cannot effectively control $\mathrm{PONV}^{[7]}$. Thus, recently

\section{Editor: Arjun Ballal.}

This work was supported by the Bio \& Medical Technology Development Program of the National Research Foundation (NRF) funded by the Korean government (MSIT) (2017M3A9D8063538)

The authors have no conflicts of interests to disclose.

The datasets generated during and/or analyzed during the current study are available from the corresponding author on reasonable request.

${ }^{a}$ Department of Orthopedic Surgery, Seoul National University College of Medicine, SMG-SNU Boramae Medical Center, ${ }^{b}$ Department of Emergency Medicine, Seoul National University College of Medicine, Seoul National University Hospital, Seoul, ' Department of Orthopedic Surgery, Seoul National University College of Medicine, Seoul National University Bundang Hospital, Seongnam-si, Gyeonggi-do, South Korea.

* Correspondence: Moon Jong Chang, Department of Orthopedic Surgery, Seoul National University College of Medicine, SMG-SNU Boramae Medical Center, 5 Gil 20, Boramae-road, Dongjak-gu, Seoul 07061, South Korea (e-mail: moonjongchang@gmail.com).

Copyright (c) 2021 the Author(s). Published by Wolters Kluwer Health, Inc.

This is an open access article distributed under the terms of the Creative Commons Attribution-Non Commercial License 4.0 (CCBY-NC), where it is permissible to download, share, remix, transform, and buildup the work provided it is properly cited. The work cannot be used commercially without permission from the journal.

How to cite this article: Park HJ, Chang MJ, Kang SB, Hwang lu, Kim JS, Chang CB. Effects of preoperative, scheduled administration of antiemetics in reducing postoperative nausea and vomiting in patients undergoing total knee arthroplasty. Medicine 2021;100:1(e24143).

Received: 21 October 2020 / Received in final form: 4 December 2020 / Accepted: 7 December 2020

http://dx.doi.org/10.1097/MD.0000000000024143 
there has been an interest in preemptive, scheduled use of antiemetic drugs for the prevention of $\mathrm{PONV}^{[8]}$.

A systematic review reported that the administration of antiemetic drugs significantly affected PONV regardless of the type of surgery ${ }^{[9]}$. However, consensus remains to be established regarding the timing of administration of antiemetic drugs in patients undergoing TKA. In previous studies, the pre-emptive antiemetics were usually administrated at the end of surgery or at the induction of anesthesia ${ }^{[7,10,11]}$. However, despite administration of the antiemetics before an emetic event, the patients were already exposed to the noxious stimuli such as stress, anesthesia, and the surgical procedure ${ }^{[12,13]}$. Additionally, not only some patients experienced motion sickness when being transported from the ward to the operating room and some people complained of nausea, which arose from the stress response of the autonomic nervous system ahead of surgery. ${ }^{[14]}$ The maximum intravenous level of ramosetron (5-HT3 receptor antagonist) is reached within 2 hours of administration and decreased by half at 6 hours after the injection. ${ }^{[15]}$ However, the efficacy of the drug was maintained at 48 hours. ${ }^{[16,17]}$ The known surgical time for primary TKA is known from 89 to 107 minutes ${ }^{[7,10,18]}$. Considering these findings, we hypothesized that the administration of ramosetron 1 hour before surgery would result in maximal level of the drug at the end of surgery and its effectiveness might be maintained until 48 hours after TKA. One study reported that preoperative use of aprepitant (NK1 receptor antagonist) 2 hours before surgery reduced the incidence of PONV after total hip arthroplasty and TKA. ${ }^{[19]}$ However, no study has reported the effectiveness of ramosetron administrated 1 hour before surgery on PONV when the events were not triggered by noxious stimuli.

Pain control after surgery is associated with PONV. There are consistent reports in the literature regarding the effects of preemptive pain management on reducing postoperative pain and PONV ${ }^{[20,21]}$ However, the effects of scheduled administration of antiemetics in reducing postoperative pain remains controversial. $^{[7,22,23]}$

The aims of this study were to determine whether preoperative scheduled administration of an antiemetic could reduce PONV after TKA compared to postoperative administration, and to determine whether there was a difference in postoperative pain, patient satisfaction, and complications after TKA between the 2 administration times. We hypothesized that preoperative administration of an antiemetic would reduce PONV compared to postoperative administration. We also hypothesized that preoperative administration of an antiemetic would reduce postoperative pain and improve patient satisfaction without increased complications compared to postoperative administration.

\section{Methods}

A total of 101 patients who underwent primary TKA due to endstage osteoarthritis from July 2015 to January 2016 at our institution were included in this study. The study was designed according to a previous study on the effects of the ramosetron in reducing PONV in patients undergoing TKA. ${ }^{[7]}$ Patients who underwent TKA $(\mathrm{N}=178)$ were assessed for eligibility for inclusion in the study. Seventy seven patients were excluded based on the proposed exclusion criteria. The inclusion criterion was the patients with primary osteoarthritis undergoing unilateral TKA. The exclusion criteria were as follows:
1. patients undergoing TKA, not due to osteoarthritis;

2. revision TKA;

3. patients undergoing bilateral TKA;

4. patients with an allergy to 5-HT3 receptor antagonists;

5 . history of other antiemetic drug use within 24 hours before surgery; and

6. history of cardiovascular or respiratory disease, alcohol or opioid dependence, and renal or hepatic failure (Fig. 1).

Approval was obtained from the Institutional Review Board of the authors hospital (IRB No.: 26-2015-16, SMG-SNU BMC) before commencement of the study. The study protocol was registered in the Clinical Research Information Service (Register number: KCT0005080). Informed consent was obtained from all participants included in this study.

There were several risk factors for PONV. ${ }^{[24]}$ Among them, 4 risk factors which included female sex, history of PONV or motion sickness, nonsmoking status, and the use of postoperative opioids, were selected to calculate the risk for PONV in the patients. The simplified risk assessment tool described by Apfel et al was used to evaluate the risk for PONV. ${ }^{[25]}$

Eligible patients either received intravenous scheduled administration of $0.3 \mathrm{mg}$ ramosetron 1 hour before surgery (preinjection group) at ward or the end of surgery (post-injection group) at operating room by registered nurses in consecutive order. The antiemetic drug was injected preoperatively in the first 50 patients, and the same drug was injected postoperatively in the subsequent 51 patients undergoing TKA. All patients received the same anesthetic and multimodal pain management protocol, excluding the timing of antiemetic drug administration. Among the antiemetic drugs currently used, 5-hydroxy-tryptamine receptor 3 (5-HT3) antagonist (e.g., ondansetron, granisetron, and ramosetron) are most commonly used to prevent PONV. We chose ramosetron, as used in a previous study, due to its increased potency and longer-acting properties than other serotonin receptor antagonists. ${ }^{[7]}$ Preemptive medication for multimodal pain management was given 1 hour preoperatively to all patients on a call basis and consisted of $200 \mathrm{mg}$ celecoxib, $75 \mathrm{mg}$ pregabalin, and $650 \mathrm{mg}$ acetaminophen. Intravenous $5 \mathrm{mg}$ dexamethasone was also used to reduce postoperative PONV. There were no differences between the 2 groups in terms of demographic data, duration of surgery, level of spinal anesthesia, the number of risk factors, and calculated mean risk for PONV (Table 1).

All surgical procedures were performed by one senior surgeon using the standard medial parapatellar arthrotomy approach with a tourniquet. Before cementation of the prosthesis, a periarticular cocktail injection, comprising $300 \mathrm{mg}$ ropivacaine, $10 \mathrm{mg}$ morphine sulfate, $30 \mathrm{mg}$ ketorolac, $300 \mu \mathrm{g}$ 1:1000 epinephrine, and $1 \mathrm{~g}$ cefazolin, was injected entirely throughout the joint capsule and synovium. After surgery, patients received intravenous patient-controlled analgesia (PCA), which was programmed to deliver $1 \mathrm{ml}$ of a $100 \mathrm{ml}$ solution containing $2000 \mu \mathrm{g}$ fentanyl for the patients younger than 70 years and $1500 \mu \mathrm{g}$ fentanyl for those older than 70 years. The device was programmed to be locked for 10 minutes if the patient pressed the button of the pump. Intramuscular injection of ketoprofen was administered for acute pain relief in case where the patientreported severity of pain level was 6 or higher on a pain visual analogue scale (VAS).

Patients were monitored for PONV for the first 6 hours, followed by every 6 hours until 24 hours, and 24 to 48 hours 


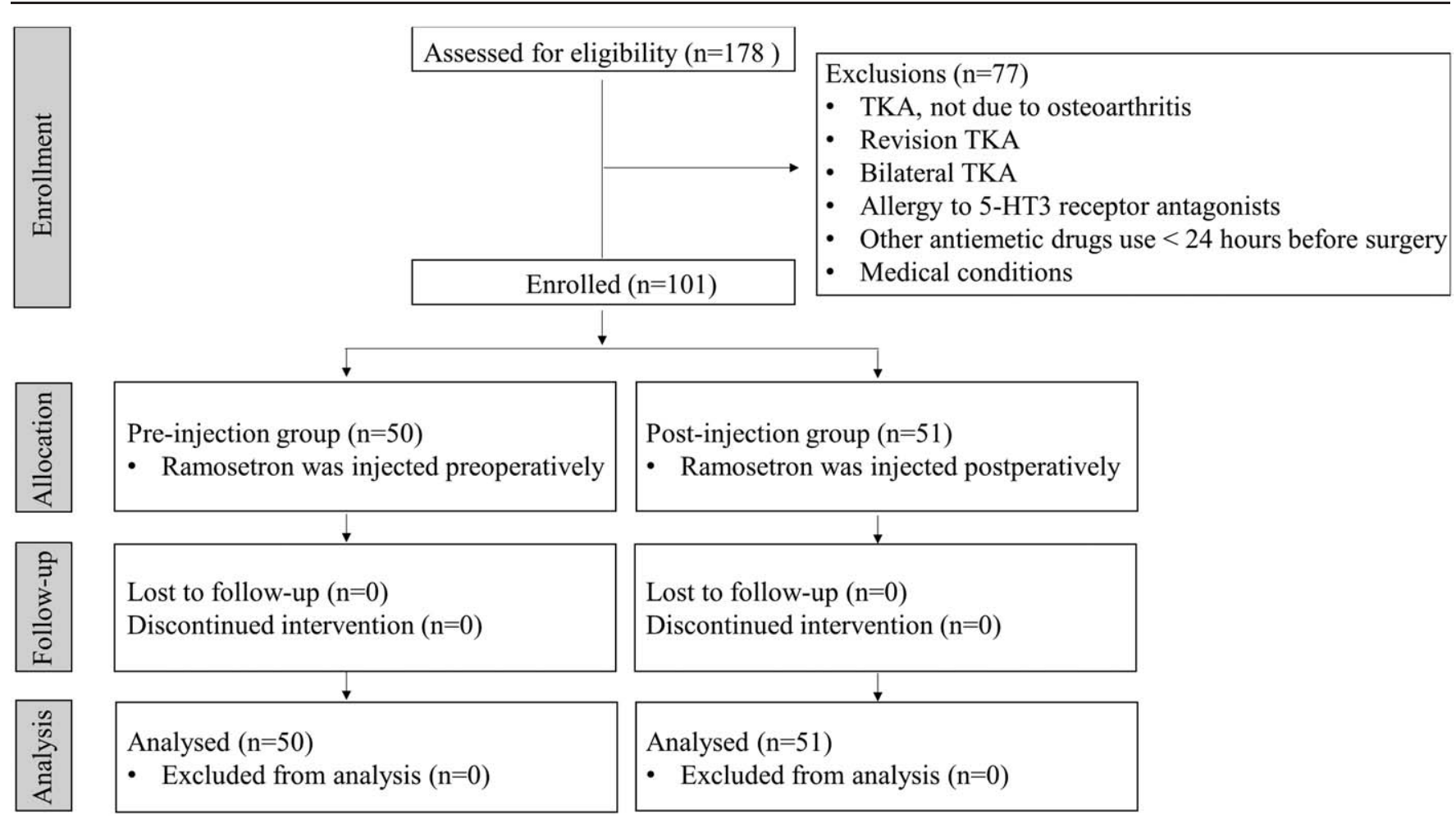

Figure 1. Flow chart for the study.

postoperatively by the same clinical investigator who did not have information on the injection method was used. Nausea was defined as the awareness of the urge to vomit or retch. The frequency and intensity of nausea were recorded. Nausea intensity was also assessed using a VAS (0-10; 0, no nausea and 10 , most severe nausea). Vomiting was defined as the

\section{Table 1}

Demographics and the risk factors for PONV.

\begin{tabular}{|c|c|c|c|}
\hline & $\begin{array}{l}\text { Preoperative } \\
\text { injection } \\
(\mathrm{N}=50)\end{array}$ & $\begin{array}{c}\text { Postperative } \\
\text { injection } \\
(\mathrm{N}=51)\end{array}$ & $P$ value \\
\hline \multicolumn{4}{|l|}{ Demographic data } \\
\hline Age (years) & $71.3 \pm 6.2$ & $71.2 \pm 5.9$ & .911 \\
\hline Proportion of female ${ }^{\mathrm{a}}$ & $43(86 \%)$ & $47(92 \%)$ & .321 \\
\hline Height (cm) & $153.2 \pm 6.7$ & $153.7 \pm 6.9$ & .666 \\
\hline Weight (kg) & $61.4 \pm 8.3$ & $64.4 \pm 10.6$ & .125 \\
\hline Body mass index $\left(\mathrm{kg} / \mathrm{m}^{2}\right)$ & $26.2 \pm 3.2$ & $27.3 \pm 4.5$ & .171 \\
\hline Laterality (Right: Left) & 22: 28 & $24: 27$ & .609 \\
\hline Duration of surgery (minutes) & $95.9 \pm 17.4$ & $94.6 \pm 12.8$ & .801 \\
\hline Level of spinal anesthesia & $\mathrm{T} 5(\mathrm{~T} 1-\mathrm{T} 12)$ & $\mathrm{T} 5(\mathrm{~T} 1-\mathrm{T} 10)$ & .553 \\
\hline \multicolumn{4}{|l|}{ Risk factors identified ${ }^{b}$} \\
\hline With one factor & 6 & 2 & .133 \\
\hline With two factors & 27 & 27 & .915 \\
\hline With three factors & 13 & 17 & .420 \\
\hline With four factors & 4 & 5 & .750 \\
\hline Calculated mean risk ${ }^{c}$ & $2.3 \pm 0.8$ & $2.5 \pm 0.7$ & .212 \\
\hline
\end{tabular}

Data are presented as numbers of patients.

${ }^{a}$ Data are presented as number of female patients and their percentages in parentheses.

${ }^{\mathrm{b}}$ The risk factors for PONV described by Murphy et al.

${ }^{\mathrm{c}}$ Data are presented as calculated mean risk for PONV based on the risk scoring system described by Apfel et al.

PONV = postoperative nausea and vomiting. expulsion of the stomach contents through the mouth, and each episode of vomiting was recorded. Rescue antiemetics were administrated to patients with a VAS score of $>4$ for nausea, an episode of vomiting, or upon their request. Intravenous injection of $10 \mathrm{mg}$ metoclopramide was used as the first-line rescue antiemetic treatment, and if severe nausea persisted after 2 consecutive boluses of metoclopramide in a 30-minute interval; $4 \mathrm{mg}$ ondansetron was administered intravenously as the secondline treatment. The VAS (0-10; 0 , very dissatisfied, 10 , very satisfied) was also used to assess patient satisfaction after 48 hours of surgery. Common adverse drug reactions associated with serotonin receptor antagonists such as headaches, dizziness, and drowsiness were also assessed and reported.

The sample size was calculated based on the description in a previous study where the incidence of PONV was 54\%. ${ }^{[7]}$ Fifty patients were required in each group to determine a $50 \%$ reduction in the incidence of PONV. Continuous variables between 2 groups such as age, height, weight, body mass index, duration of surgery, calculated mean risk, VAS scores were analyzed by the Student $t$ test. Chi-Squared or Fisher exact tests were used for categorical variables such as sex, risk factor for PONV, incidence of emetic events and frequency of antiemetic rescue medicine use. All analyses were performed with SPSS for Windows version 26.0 (SPSS Inc, Chicago, IL). $P$ value of $<.05$ was considered statistically significant.

\section{Results}

Preoperative administration of ramosetron did not decrease PONV during the first 48 hours. Although there was a tendency of reduced incidence of nausea in the preoperative injection group compared to the postoperative injection group (62\% vs $86 \%$, 
Table 2

Incidence of emetic events and use of rescue antiemetics in Preoperative injection and Postinjection group.

\begin{tabular}{lccc}
\hline & $\begin{array}{c}\text { Preoperative } \\
\text { injection (N=50) }\end{array}$ & $\begin{array}{c}\text { Postperative } \\
\text { injection (N=51) }\end{array}$ & $\boldsymbol{P}$ value \\
\hline Nausea & & & \\
$\quad$ For 48 hours & $31(62 \%)$ & $43(86 \%)$ & .075 \\
0-6 hours & 11 & 10 & .767 \\
6-24 hours & 14 & 21 & .164 \\
24-48 hours & 6 & 12 & .130 \\
Vomiting & & & \\
$\quad$ For 48 hours & $7(14 \%)$ & $9(18 \%)$ & .846 \\
0-6 hours & 1 & 3 & .317 \\
6-24 hours & 4 & 5 & .750 \\
24-48 hours & 2 & 1 & .546 \\
Rescue antiemetics & & & .596 \\
For 48 hours & $8(16 \%)$ & $6(12 \%)$ & .317 \\
0-6 hours & 1 & 3 & .630 \\
6-24 hours & 3 & 2 & .162 \\
24-48 hours & 4 & 1 & \\
\hline
\end{tabular}

Data are presented as the numbers of incidence and the percentage in the parentheses.

respectively, $P=.075$ ), there was no significant difference between the 2 groups in terms of incidence of nausea and vomiting events, use of rescue antiemetics, and the severity of nausea $(P>.05)$ (Tables 2 and 3 ).

Although there was no significant difference in the incidence of complications related to ramosetron based on the timing of administration, preoperative administration of ramosetron did not reduce postoperative pain or improve patient satisfaction. There was no difference in the consumption of fentanyl and the severity of postoperative pain between the 2 groups $(P>.05$ in all postoperative periods). Additionally, there was no significant difference in patient satisfaction $(1.7 \pm 2.3$ vs $2.0 \pm 2.2$, respectively, $P>.05)$ and the incidence of complications related to ramosetron between the 2 groups $(P>.05)$ (Table 4$)$.

\section{Discussion}

Reducing the incidence of PONV after TKA is important to improve patient recovery and shorten the LOS. ${ }^{[7]}$ Additionally, it is important to increase patient satisfaction and optimize postoperative fluid management by initiating oral intake as soon as possible after surgery. Several methods have been used to decrease PONV, including reduced use of opioids with multimodal pain management, systemic steroid use, and scheduled use or continuative use of antiemetics. ${ }^{[8,26]}$ However, among these, the effect of the timing of use of antiemetics remains controversial. The principal finding of this study was that

\section{Table 3}

Comparison of the severity of nausea between Preoperative injection and Postinjection group.

\begin{tabular}{lccr}
\hline & $\begin{array}{c}\text { Preoperative } \\
\text { injection (N=50) }\end{array}$ & $\begin{array}{c}\text { Postperative } \\
\text { injection (N=51) }\end{array}$ & $\boldsymbol{P}$ value \\
\hline 0-6hours & $0.9 \pm 1.8$ & $1.0 \pm 2.4$ & .705 \\
6-24 hours & $1.4 \pm 2.4$ & $1.8 \pm 2.7$ & .307 \\
24-48 hours & $0.7 \pm 2.1$ & $0.8 \pm 1.6$ & .776 \\
\hline
\end{tabular}

Data are presented as means with standard deviation using VAS. VAS = visual analogue scale
Table 4

Pain score, satisfaction score and the incidence of complications.

\begin{tabular}{lccc}
\hline & $\begin{array}{c}\text { Preoperative } \\
\text { injection (N=50) }\end{array}$ & $\begin{array}{c}\text { Postoperative } \\
\text { injection (N=51) }\end{array}$ & $\boldsymbol{P}$ value \\
\hline Pain score (VAS) & & & \\
0-6 hours & $5.7 \pm 1.8$ & $5.1 \pm 1.8$ & .092 \\
6-24 hours & $6.8 \pm 1.8$ & $6.5 \pm 2.1$ & .455 \\
24-48 hours & $6.2 \pm 1.7$ & $5.5 \pm 2.1$ & .081 \\
Fentanyl consumption ( $\mu \mathrm{g})$ & & & \\
For 48 hours & $59.9 \pm 14.1$ & $51.0 \pm 23.2$ & .066 \\
0-6 hours & $16.5 \pm 6.1$ & $14.0 \pm 7.6$ & .335 \\
6-24 hours & $24.3 \pm 10.7$ & $21.2 \pm 13.2$ & .346 \\
24-48 hours & $19.1 \pm 9.2$ & $15.8 \pm 11.0$ & .182 \\
Satisfaction score (VAS) & $1.7 \pm 2.3$ & $2.0 \pm 2.2$ & .561 \\
Headache & & & \\
For 48 hours & $15(30 \%)$ & $17(33.3 \%)$ & .584 \\
0-6 hours & 9 & 7 & .556 \\
6-24 hours & 6 & 9 & .425 \\
24-48 hours & 0 & 1 & 1.000 \\
Dizziness & & & \\
For 48 hours & $17(34 \%)$ & $21(41.2 \%)$ & .692 \\
0-6 hours & 9 & 9 & .963 \\
6-24 hours & 7 & 11 & .320 \\
24-48 hours & 1 & 1 & 1.000 \\
Drowsiness & & & \\
For 48 hours & $14(28 \%)$ & $11(21.6 \%)$ & .376 \\
0-6 hours & 7 & 5 & .515 \\
6-24 hours & 6 & 3 & .318 \\
24-48 hours & 1 & 3 & .617 \\
\hline
\end{tabular}

Data are presented as the numbers of incidence and the percentage in the parentheses. VAS = visual analogue scale.

preoperative administration of the antiemetic did not reduce postoperative nausea, vomiting, and pain compared to postoperative administration. In addition, preoperative use of the antiemetic was not associated with improvement in patient satisfaction.

Our findings negated the hypothesis that preoperative scheduled administration, rather than postoperative administration, of an antiemetic would reduce PONV after TKA. One study reported that ramosetron administrated 30 minutes before the end of surgery decreased the incidence of nausea in the 2- to 6hour and the 6- to 24 -hour postoperative period. ${ }^{[10]}$ However, in our study, administration of ramosetron 1 hour before surgery did not reduce PONV in the patients until 48 hours after the surgery compared to those in the post-injection group. This could be attributed to the other medications used, such as celecoxib, pregabalin, acetaminophen, as well as dexamethasone, to control postoperative pain, nausea and vomiting. However, dexamethasone was not used in the previous study for controlling PONV and there was no description of multimodal pain management such as premedication and periarticular injection (PAI). Maximal blood levels of dexamethasone are achieved 1.6 to 2 hours after the injection. Therefore, patients who received dexamethasone were at lower risk of experiencing POVN compared to the patients who did not receive the drug. ${ }^{[27]}$ This discrepancy could be partly explained by the difference in multimodal medication. Thus, although there was a tendency to reduce the incidence of nausea for 48 hours postoperatively, the effectiveness might not have been significant when the patients were managed with a different multimodal therapy for postoperative pain, nausea and vomiting. 
Our findings negated our hypothesis that preoperative administration of an antiemetic would reduce postoperative pain and improve patient satisfaction. In instances where the pain after surgery was not controlled, the patients required more pain medications including opioids, which caused nausea and vomiting. Therefore, postoperative pain control is important to manage the incidence of PONV. However, the effect of antiemetic on postoperative pain control has not been established. ${ }^{[7,22,23,28,29]}$ One study reported that ramosetron reduced the severity of postoperative pain after thyroid surgery. ${ }^{[23]}$ Another study reported that ramosetron was superior to ondansetron for postoperative pain control 24 to 48 hours after lumbar spine surgery ${ }^{[22]}$. On the other hand, Koh et al reported that ramosetron did not provide postoperative pain relief after TKA. ${ }^{[7]}$ Hartrick et al reported that antiemetic (aprepitant) did not decrease the pain after TKA. The findings of our study were line with those reported by Koh et al and Hartrick et al Early use of ramosetron was not advantageous in reducing the severity of pain and consumption of fentanyl. Patients undergoing TKA, unlike those undergoing thyroid or spine surgery, generally receive premedication and PAIs for pain management. The type of surgery and pain control protocol, such as premedication and PAI might contribute to the effectiveness of ramosetron in postoperative pain control. Based on the similar rate of complications and absence of any benefit in controlling postoperative pain, nausea and vomiting, the lack of improvement in patient satisfaction was comprehensible after surgery in the pre-injection group. Preoperative administration of ramosetron did not present any clinical advantage, as there was no significant difference in the incidence of postoperative nausea, vomiting, pain, and complications. However, given the different circumstances of each hospital, preoperative administration of ramosetron might be one of the alternatives that could be considered to reduce the incidence of PONV.

Our study has several limitations. First, the proportion of female included in the study was high. Therefore, there is a possibility of different results when it applied to the population with different sex proportion. Second, the patients included in this study were not randomly assigned to each group. However, we sequentially used the 2 different methods for scheduled administration of the antiemetic in patients undergoing TKA with similar demographic characteristics. Thus, in our opinion, the selection bias was minimized. Third, all patients received an intravenous injection of dexamethasone. Dexamethasone has a substantial role in reducing PONV; thus, it could affect the incidence of PONV after TKA. ${ }^{[30]}$ Fourth, our study compared the timing of antiemetic usage but did not include a placebo control group. Fifth, we used ramosetron alone as the primary antiemetic. Finally, multimodal therapies were used in all patients in our study to control PONV. However, the results obtained could differ after administration of ramosetron, in cases where multimodal therapies for controlling PONV was not given to patients. Pharmacological characteristics of different antiemetic drugs may vary. Therefore, our findings would not be applicable to other antiemetics with different pharmacological characteristics.

\section{Conclusion}

Preoperative scheduled administration of ramosetron showed a modest effect in reducing the incidence of postoperative nausea compared to postoperative administration of the drug. However, the difference observed was not clinically significant. Under the current protocol of multimodal therapies, timing of administration of preemptive antiemetics did not have significant effect on PONV.

\section{Acknowledgments}

We acknowledged So Hee Oh, Ph D for advice on statistical analysis.

\section{Author contributions}

Conceptualization: Hyung Jun Park, Moon Jong Chang, SeungBaik Kang, Il-ung Hwang, Jong Seop Kim, Chong Bum Chang. Investigation: Hyung Jun Park, Chong Bum Chang.

Methodology: Hyung Jun Park, Moon Jong Chang, Chong Bum Chang.

Supervision: Moon Jong Chang, Seung-Baik Kang, Chong Bum Chang.

Validation: Il-ung Hwang, Jong Seop Kim.

Writing - original draft: Hyung Jun Park, Moon Jong Chang, Chong Bum Chang.

Writing - review \& editing: Hyung Jun Park, Moon Jong Chang, Chong Bum Chang.

\section{References}

[1] Macario A, Weinger M, Carney S, et al. Which clinical anesthesia outcomes are important to avoid? The perspective of patients. Anesth Analg 1999;89:652-8.

[2] Capdevila X, Barthelet Y, Biboulet P, et al. Effects of perioperative analgesic technique on the surgical outcome and duration of rehabilitation after major knee surgery. Anesthesiology 1999;91:8-15.

[3] Cole PJ, Craske DA, Wheatley RG. Efficacy and respiratory effects of low-dose spinal morphine for postoperative analgesia following knee arthroplasty. Br J Anaesth 2000;85:233-7.

[4] Makaryus R, Miller TE, Gan TJ. Current concepts of fluid management in enhanced recovery pathways. Br J Anaesth 2018; 120:376-83.

[5] Dorr LD, Chao L. The emotional state of the patient after total hip and knee arthroplasty. Clin Orthop Relat Res 2007;463:7-12.

[6] Myles PS, Williams DL, Hendrata M, et al. Patient satisfaction after anaesthesia and surgery: results of a prospective survey of 10,811 patients. Br J Anaesth 2000;84:6-10.

[7] Koh IJ, Chang CB, Jeon YT, et al. Does ramosetron reduce postoperative emesis and pain after TKA? Clin Orthop Relat Res 2012;470:1718-27.

[8] Antle O, Kenny A, Meyer J, et al. Antiemetics for postoperative nausea and vomiting in patients undergoing elective arthroplasty: scheduled or as needed? Can J Hosp Pharm 2019;72:139-44.

[9] Ahn E, Choi G, Kang H, et al. Palonosetron and ramosetron compared for effectiveness in preventing postoperative nausea and vomiting: a systematic review and meta-analysis. PLoS One 2016;11:e0168509.

[10] Hahm T, Ko J, Choi S, et al. Comparison of the prophylactic anti-emetic efficacy of ramosetron and ondansetron in patients at high-risk for postoperative nausea and vomiting after total knee replacement. Anaesthesia 2010;65:500-4.

[11] Ryu J-H, Jeon Y-T, Min B, et al. Effects of palonosetron for prophylaxis of postoperative nausea and vomiting in high-risk patients undergoing total knee arthroplasty: A prospective, randomized, double-blind, placebo-controlled study. PloS One 2018;13.

[12] Chatterjee S, Rudra A, Sengupta S. Current concepts in the management of postoperative nausea and vomiting. Anesthesiol Res Pract 2011;2011.

[13] Jabalameli M, Honarmand A, Safavi M, et al. Treatment of postoperative nausea and vomiting after spinal anesthesia for cesarean delivery: a randomized, double-blinded comparison of midazolam, ondansetron, and a combination. Advanced Biomed Res 2012;1.

[14] Ziegler MG. Psychological stress and the autonomic nervous system. Primer on the Autonomic Nervous System 2012;Elsevier, 291-293.

[15] Nakajima MJJJCEM. Phase I study of YM060 tablets in healthy subjects. Single intravenous dose study 1994;71:2461-8. 
Park et al. Medicine (2021) 100:1

Medicine

[16] Fujii Y, Tanaka H, Kawasaki T. Benefits and risks of granisetron versus ramosetron for nausea and vomiting after breast surgery: a randomized, double-blinded, placebo-controlled trial. Am J Therapeutics 2004; 11:278-82.

[17] Rabasseda X. Ramosetron, a 5-HT3 receptor antagonist for the control of nausea and vomiting. Drugs of Today, 38 Spain: Barcelona; 2002. 75-89.

[18] Young SW, Mutu-Grigg J, Frampton CM, et al. Does speed matter? Revision rates and functional outcomes in TKA in relation to duration of surgery. J Arthroplasty 2014;29:1473-7. ex.

[19] Dilorio TM, Sharkey PF, Hewitt AM, et al. Antiemesis after total joint arthroplasty: does a single preoperative dose of aprepitant reduce nausea and vomiting? Chin Orthopaed Related Research ${ }^{\circledR}$ 2010; 468:2405-9.

[20] Kob IJ, Chang CB, Lee JH, et al. Preemptive low-dose dexamethasone reduces postoperative emesis and pain after TKA: a randomized controlled study. Chin Orthopaed Related Research ${ }^{\circledR}$ 2013;471: 3010-20.

[21] Lee JK, Chung K-S, Choi CH. The effect of a single dose of preemptive pregabalin administered with COX -2 inhibitor: a trial in total knee arthroplasty. J Arthroplasty 2015;30:38-42.

[22] Choi YS, Shim JK, Jeon DH, et al. Effect of ramosetron on patientcontrolled analgesia related nausea and vomiting after spine surgery in highly susceptible patients: comparison with ondansetron. Spine 2008;33:E602-6.
[23] Song Y-K, Lee C. Effects of ramosetron and dexamethasone on postoperative nausea, vomiting, pain, and shivering in female patients undergoing thyroid surgery. J Anesth 2013;27:29-34.

[24] Murphy MJ, Hooper VD, Sullivan E, et al. Identification of risk factors for postoperative nausea and vomiting in the perianesthesia adult patient. J Perianesth Nurse 2006;21:377-84.

[25] Appel CC, Läärä E, Koivuranta M, et al. A simplified risk score for predicting postoperative nausea and vomiting conclusions from crossvalidations between two centers. Anesthesiology 1999;91:693.

[26] Jung HS, Huh J, Kim Y, et al. Continuous administration of ramosetron with patient-controlled analgesia after laparoscopic distal gastrectomy does not delay postoperative bowel function recovery: a prospective, randomized, double-blinded study. Medicine 2018;97:e11503.

[27] Low D, Schuster O, Gaul E. Dose-dependent pharmacokinetics of dexamethasone. Europ J Chin Pharmacol 1986;30:225-30.

[28] Hartrick CT, Tang YS, Hunstad D, et al. Aprepitant vs. Multimodal prophylaxis in the prevention of nausea and vomiting following extended-release epidural morphine. Pain Practice 2010;10:245-8.

[29] Jellish WS, Leonetti JP, Kristina S, et al. Morphine/ondansetron PCA for postoperative pain, nausea, and vomiting after skull base surgery. Otolaryngol Head Neck Surg 2006;135:175-81.

[30] Award K, Ahmed H, Abushouk AI, et al. Dexamethasone combined with other antiemetics versus single antiemetics for prevention of postoperafive nausea and vomiting after laparoscopic cholecystectomy: an updated systematic review and meta-analysis. Int J Surg 2016;36:152-63.

6 